\title{
Calibration error analysis of inertially stabilized platforms using quaternions and octonions in rotation decomposition
}

Proc IMechE Part B:

J Engineering Manufacture 2016, Vol. 230(9) I77|-1777 (c) IMechE 2015

Reprints and permissions: sagepub.co.uk/journalsPermissions.nav DOI: $10.1177 / 0954405415611366$ pib.sagepub.com @SAGE

\author{
Qijian Tang ${ }^{1,2,3}$, Xiangjun Wang', Qingping Yang ${ }^{3}$ and Feng Liu'
}

\begin{abstract}
In the calibration process of the inertially stabilized platforms with a high-precision turntable and an autocollimator, significant calibration errors can result from the axis misalignments between the inertially stabilized platforms and the turntable. Based on the relationship between spatial rotations and quaternions or octonions, this article proposes a representation using octonions to realize the decomposition of the rotation axis in two perpendicular axes and subsequently derives the calibration error model. The test results demonstrated that the error is significantly improved after compensation. The azimuth variance is reduced from $0.1379\left({ }^{\circ}\right)^{2}$ to $0.0492\left({ }^{\circ}\right)^{2}$, which offers a more accurate set of data for further compensation based on the error model of the platform itself.
\end{abstract}

\section{Keywords}

Rotation, quaternion, octonion, spinor, inertially stabilized platform calibration

Date received: 25 June 2015; accepted: 21 September 2015

\section{Introduction}

As a vector rotates around a straight line in a threedimensional (3D) space coordinate system, it is easy to obtain the rotated vector through a rotation matrix. ${ }^{1-5}$ Inertially stabilized platforms (ISPs) have been widely utilized to maintain its sensor's orientation pointing to an accurate direction in many applications, such as vehicles, ships, aircrafts and spacecraft. Its accuracy is technically vital and affects the capture, location and tracking of the target. ${ }^{6-9}$ A two-axial platform is fixed on a high-precision turntable, with an autocollimator showing the rotating angular error, ${ }^{10,11}$ as shown in Figure 1 , where $A_{1}$ is the azimuth axis of the turntable, $B_{1}$ its elevation axis, $A_{2}$ the azimuth axis of the platform, $B_{2}$ its elevation axis and $C_{2}$ the line of sight. At zero positions, $A_{1}$ and $A_{2}$ are vertical, while $B_{1}$ and $B_{2}$ are horizontal.

In the calibration process, $\theta_{P A}$ and $\theta_{P E}$ are the platform rotation angles in terms of azimuth and elevation, respectively. The azimuth and elevation angles can be similarly defined for the turntable $\left(\theta_{T A}\right.$ and $\left.\theta_{T E}\right)$ and the autocollimator readouts of axes $\left(\delta_{A}\right.$ and $\left.\delta_{E}\right)$. Generally, it is $\theta_{T A}=-\theta_{P A}$ and $\theta_{T E}=-\theta_{P E}$. If $\theta_{P E}=0$ and $\theta_{T E}=0$, it is obvious that $\delta_{A}=\theta_{P A}-\theta_{T A}$. However, if $\theta_{T E}=-\theta_{P E} \neq 0, \mathrm{~A}_{1}$ and $\mathrm{A}_{2}$ are no longer vertical, which indicates $\delta_{A} \neq \theta_{P A}-\theta_{T A}$. It means some calibration error is introduced, and it is obligatory to determine the error to calibrate ISPs accurately.

This article first presents the notation and definitions of quaternions and octonions and then gives a detailed description of the relationship between rotations and quaternions or octonions. It obtains the spinor with octonions as a vector rotates around an axis by means of decomposing the shaft into two perpendicular axes, which indicates octonions can also be used in rotations. The actual test results have demonstrated that the calibration process error can be effectively compensated.

\footnotetext{
'MOEMS Education Ministry Key Laboratory, Tianjin University, Tianjin, P.R. China

${ }^{2}$ Key Laboratory of Optoelectronic Devices and Systems of Ministry of Education and Guangdong Province, Shenzhen University, Shenzhen, P.R. China

${ }^{3}$ College of Engineering, Design and Physical Sciences, Brunel University London, Uxbridge, UK
}

\section{Corresponding author:}

Feng Liu, MOEMS Education Ministry Key Laboratory, Tianjin University, Tianjin 300072, P.R. China.

Email: tjuliufeng@tju.edu.cn 


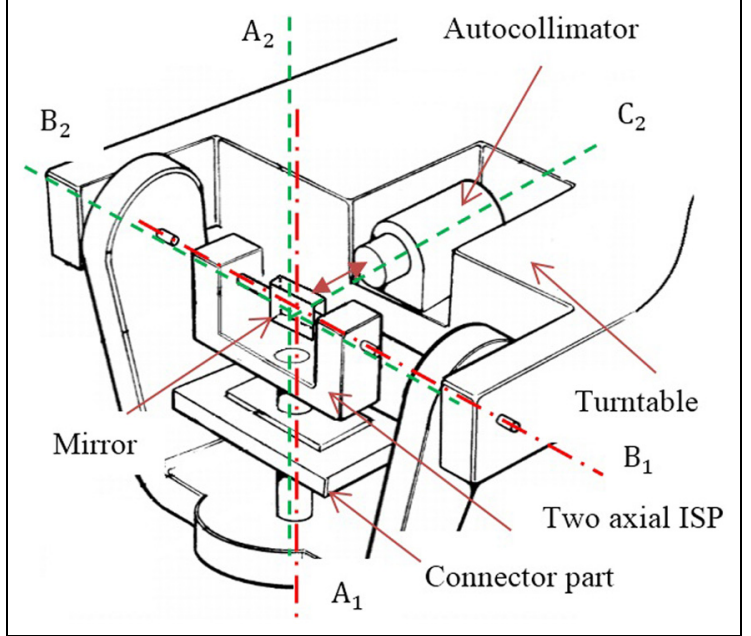

Figure I. Calibration system of ISPs.

\section{Quaternions and octonions}

\section{Notation and definitions of quaternions}

Quaternions are invented by W.R. Hamilton, and they can be used to speed up calculations involving rotations. A quaternion is represented by just four scalars, in contrast to a $3 \times 3$ rotation matrix which has nine scalar entries. ${ }^{12}$ A quaternion can be defined as ${ }^{13}$

$$
\mathrm{Q}=\mathrm{a} \boldsymbol{i}+\mathrm{b} \boldsymbol{j}+\mathrm{c} \boldsymbol{k}+\mathrm{q}_{0}
$$

where a, b, c and $\mathrm{q}_{0}$ are real numbers, and $\boldsymbol{i}, \boldsymbol{j}$ and $\boldsymbol{k}$ are the imaginary units which obey the following multiplication rules

$$
\left\{\begin{array}{c}
\boldsymbol{i}^{2}=\boldsymbol{j}^{2}=\boldsymbol{k}^{2}=-1 \\
\boldsymbol{i} \boldsymbol{j}=-\boldsymbol{j i}=\boldsymbol{k} \\
\boldsymbol{k} \boldsymbol{i}=-\boldsymbol{i k}=\boldsymbol{j} \\
\boldsymbol{j} \boldsymbol{k}=-\boldsymbol{k} \boldsymbol{j}=\boldsymbol{i}
\end{array}\right.
$$

Let $\boldsymbol{q}=\mathrm{a} \boldsymbol{i}+\mathrm{b} \boldsymbol{j}+\mathrm{c} \boldsymbol{k}$, equation (1) can then be rewritten as

$$
\mathrm{Q}=\mathrm{q}_{0}+\boldsymbol{q}
$$

where $\mathrm{q}_{0}$ denotes the scalar part, and $\boldsymbol{q}$ denotes the vector part.

Given another quaternion $\mathrm{P}\left(\mathbf{P}=\mathrm{p}_{0}+\boldsymbol{p}\right)$, their product according to the algebraic rules of multiplications given above is ${ }^{13}$

$$
\begin{aligned}
\mathrm{PQ} & =\left(\mathrm{p}_{0}+\boldsymbol{p}\right)\left(\boldsymbol{q}_{0}+\boldsymbol{q}\right) \\
& =\mathrm{p}_{0} \mathrm{q}_{0}-\boldsymbol{p} \cdot \boldsymbol{q}+\mathrm{p}_{0} \boldsymbol{q}+\mathrm{q}_{0} \boldsymbol{p}+\boldsymbol{p} \times \boldsymbol{q}
\end{aligned}
$$

where $\boldsymbol{p} \cdot \boldsymbol{q}$ and $\boldsymbol{p} \times \boldsymbol{q}$ represent the standard inner and cross products, respectively. Meanwhile, $\left(\mathrm{p}_{0} \mathrm{q}_{0}-\boldsymbol{p} \cdot \boldsymbol{q}\right)$ is the product's scalar part, and $\left(\mathrm{p}_{0} \boldsymbol{q}+\mathrm{q}_{0} \boldsymbol{p}+\boldsymbol{p} \times \boldsymbol{q}\right)$ is the vector part.

Assume $\mathrm{p}_{0}=0$ and $\mathrm{q}_{0}=0$, thus $\mathrm{P}=\boldsymbol{p}$ and $\mathrm{Q}=\boldsymbol{q}$ are pure quaternions. Then, equation (3) becomes

$$
\mathrm{PQ}=-\boldsymbol{p} \cdot \boldsymbol{q}+\boldsymbol{p} \times \boldsymbol{q}
$$

The norm of the quaternion $\mathrm{Q}$ is defined as in equation (5). If the norm is 1 , the quaternion is called a unit quaternion

$$
\|\mathrm{Q}\|=\sqrt{\mathrm{q}_{0}^{2}+|\boldsymbol{q}|^{2}}=\sqrt{\mathrm{q}_{0}^{2}+\mathrm{a}^{2}+\mathrm{b}^{2}+\mathrm{c}^{2}}
$$

The quaternion $\mathrm{Q}^{*}=\mathrm{q}_{0}-\boldsymbol{q}$ is called the conjugate of Q. Hence

$$
\begin{aligned}
& \mathrm{Q}^{*} \mathrm{Q}=\mathrm{QQ}^{*}=\|\mathrm{Q}\|^{2} \\
& (\mathrm{PQ})^{*}=\mathrm{Q}^{*} \mathrm{P}^{*} \\
& \|\mathrm{PQ}\|=\|\mathrm{P}\|\|\mathrm{Q}\|
\end{aligned}
$$

An arbitrary quaternion $Q$ can be described as ${ }^{14}$

$$
\mathrm{Q}=\|\mathrm{Q}\|(\cos \theta+\boldsymbol{n} \sin \theta)
$$

where $\boldsymbol{n}$ is the unit vector, and $\boldsymbol{n}=\boldsymbol{q} /\|\boldsymbol{q}\|$, $\cos \theta=\mathrm{q}_{0} /\|\mathrm{Q}\|$ and $0 \leqslant \theta<\pi$.

\section{Quaternions and rotations}

Let $S$ be an arbitrary inertial reference frame with $\boldsymbol{i}, \boldsymbol{j}$ and $\boldsymbol{k}$ as basic vectors and $\mathrm{R}_{\mathrm{s}}$ be an arbitrary vector. The unit quaternion $\mathrm{Q}$ given in quation (10) can represent a counterclockwise rotation which rotates an angle $2 \theta$ around the vector $\boldsymbol{n}$ in the coordinate system ${ }^{14}$

$$
\mathrm{Q}=\cos \theta+\boldsymbol{n} \sin \theta
$$

The resulting vector $\mathrm{R}_{\mathrm{s}}^{\prime}$ can be computed through rotation transformation by equation (11)

$$
\mathrm{R}_{\mathrm{s}}^{\prime}=\mathrm{QR}_{\mathrm{s}} \mathrm{Q}^{*}
$$

where $R^{\prime}{ }_{S}$ is the rotated vector.

Proof. As shown in Figure 2, the vector $\boldsymbol{p}$ rotates around the line $l$ at an angle $2 \theta$ to $\boldsymbol{q}$. In order to obtain $\boldsymbol{q}, \boldsymbol{p}$ can be decomposed into $\boldsymbol{p}_{\|}$which is aligned with $l$ and $\boldsymbol{p}_{\perp}$ which is perpendicular to $l$. $\boldsymbol{p}_{\perp}$ turns into $\boldsymbol{p}_{\perp}^{\prime}$ after rotation, and $\boldsymbol{p}_{\|}$remains the same, while $\boldsymbol{e}$ is the unit vector of $\boldsymbol{p}_{\|}$. Hence ${ }^{15}$

$$
\begin{aligned}
& \left\{\begin{array}{l}
\boldsymbol{p}=\boldsymbol{p}_{\|}+\boldsymbol{p}_{\perp} \\
\boldsymbol{q}=\boldsymbol{p}_{\|}+\boldsymbol{p}_{\perp}^{\prime}
\end{array}\right. \\
& \begin{aligned}
\boldsymbol{p}_{\perp}^{\prime} \boldsymbol{p}_{\perp} & =-\boldsymbol{p}_{\perp} \cdot \boldsymbol{p}_{\perp}^{\prime}-\boldsymbol{p}_{\perp} \times \boldsymbol{p}_{\perp}^{\prime} \\
& =-\left\|\boldsymbol{p}_{\perp}\right\|\left\|\boldsymbol{p}^{\prime}{ }_{\perp}\right\| \cos 2 \theta-\boldsymbol{e}\left\|\boldsymbol{p}_{\perp}\right\|\left\|\boldsymbol{p}_{\perp}{ }_{\perp}\right\| \sin 2 \theta
\end{aligned}
\end{aligned}
$$

where $\boldsymbol{p}_{\perp}, \boldsymbol{p}_{\perp}^{\prime}$ and $\boldsymbol{e}$ comply with right-handed coordinate system principle. It then has

$$
\begin{aligned}
\boldsymbol{p}_{\perp}^{\prime} & =\left(-\left\|\boldsymbol{p}_{\perp}\right\|\left\|\boldsymbol{p}_{\perp}{ }_{\perp}\right\| \cos 2 \theta-\boldsymbol{e}\left\|\boldsymbol{p}_{\perp}\right\|\left\|\boldsymbol{p}_{\perp}{ }_{\perp}\right\| \sin 2 \theta\right) \boldsymbol{p}_{\perp}^{-1} \\
& =(\cos 2 \theta+\boldsymbol{e} \sin 2 \theta) \boldsymbol{p}_{\perp}
\end{aligned}
$$

It is easy to know that

$\boldsymbol{p}_{\|}=(\boldsymbol{p} \cdot \boldsymbol{e}) \boldsymbol{e}=\frac{-1}{2}(\boldsymbol{p} \boldsymbol{e}+\boldsymbol{e p}) \boldsymbol{e}=\frac{1}{2}(\boldsymbol{p}-\boldsymbol{e p e})$ 


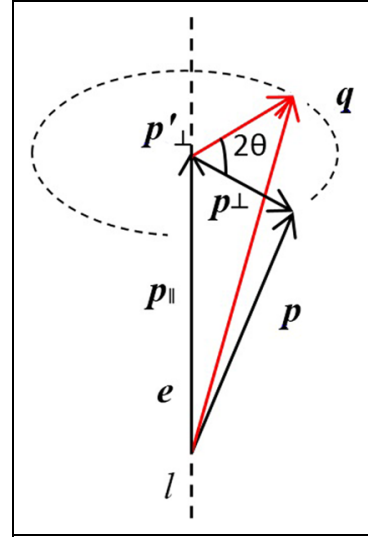

Figure 2. Schematic diagram of rotation.

Table I. Octonion multiplication table.

\begin{tabular}{|c|c|c|c|c|c|c|c|c|}
\hline & $I$ & $i$ & $j$ & $k$ & I & il & l & kl \\
\hline$I$ & $I$ & $i$ & $j$ & $k$ & $I$ & il & از & $k$ \\
\hline i & $i$ & $-I$ & $k$ & $-\boldsymbol{j}$ & il & $-I$ & $-\mathbf{k l}$ & ا \\
\hline$j$ & $\boldsymbol{j}$ & $-k$ & $-I$ & $i$ & از & kl & $-I$ & $-i l$ \\
\hline$k$ & $k$ & $j$ & $-i$ & $-I$ & $\mathbf{k l}$ & | & il & $-I$ \\
\hline I & I & $-i l$ & $-\boldsymbol{j l}$ & $-\mathbf{k l}$ & $-I$ & $i$ & $j$ & k \\
\hline il & il & $I$ & $-\mathbf{k l}$ & jl & $-\boldsymbol{i}$ & $-I$ & $-k$ & $j$ \\
\hline از & از & kl & I & $-i l$ & $-\boldsymbol{j}$ & $k$ & $-I$ & $-i$ \\
\hline kl & kl & $-j l$ & il & $I$ & $-k$ & $-\boldsymbol{j}$ & $i$ & $-I$ \\
\hline
\end{tabular}

$$
\boldsymbol{p}_{\perp}=\boldsymbol{p}-\boldsymbol{p}_{\|}=\frac{1}{2}(\boldsymbol{p}+\boldsymbol{e p e})
$$

Combined with equations (12), (14), (15) and (16), we obtain

$$
\begin{aligned}
\boldsymbol{q} & =\boldsymbol{p}_{\|}+\boldsymbol{p}_{\perp}^{\prime} \\
& =\frac{1}{2}[(1+\cos 2 \theta) \boldsymbol{p}+\sin 2 \theta \boldsymbol{e} \boldsymbol{p}-\sin 2 \theta \boldsymbol{p} \boldsymbol{e}+(\cos 2 \theta-1) \boldsymbol{e p e}] \\
& =(\cos \theta+\boldsymbol{e} \sin \theta) \boldsymbol{p}(\cos \theta-\boldsymbol{e} \sin \theta)
\end{aligned}
$$

which is in accord with equation (11); thus, quaternions can be used to describe the rotations.

\section{Definitions of octonions}

Similar to quaternions, the octonions are an eightdimensional (8D) algebra with bases $1, \boldsymbol{i}, \boldsymbol{j}, \boldsymbol{k}, \boldsymbol{l}, \boldsymbol{i l}, \boldsymbol{j} \boldsymbol{l}$ and $\boldsymbol{k} \boldsymbol{l}$, with their multiplications given in Table 1, which gives a detailed description of the multiplying results. ${ }^{16}$

It is obvious that the octonions are nonassociative since $(\boldsymbol{i j}) \boldsymbol{l}=-\boldsymbol{i}(\boldsymbol{j l})=\boldsymbol{k} \boldsymbol{l} \neq \boldsymbol{i}(\boldsymbol{j} \boldsymbol{l})$. An arbitrary octonion can be described as in equation (18)

$$
\mathrm{O}=\mathrm{a}_{0}+\mathrm{a}_{1} \boldsymbol{i}+\mathrm{a}_{2} \boldsymbol{j}+\mathrm{a}_{3} \boldsymbol{k}+\mathrm{a}_{4} \boldsymbol{l}+\mathrm{a}_{5} \boldsymbol{i} \boldsymbol{l}+\mathrm{a}_{6} \boldsymbol{j} \boldsymbol{l}+\mathrm{a}_{7} \boldsymbol{k} \boldsymbol{l}
$$

where $\mathrm{a}_{0}-\mathrm{a}_{7}$ are real numbers.

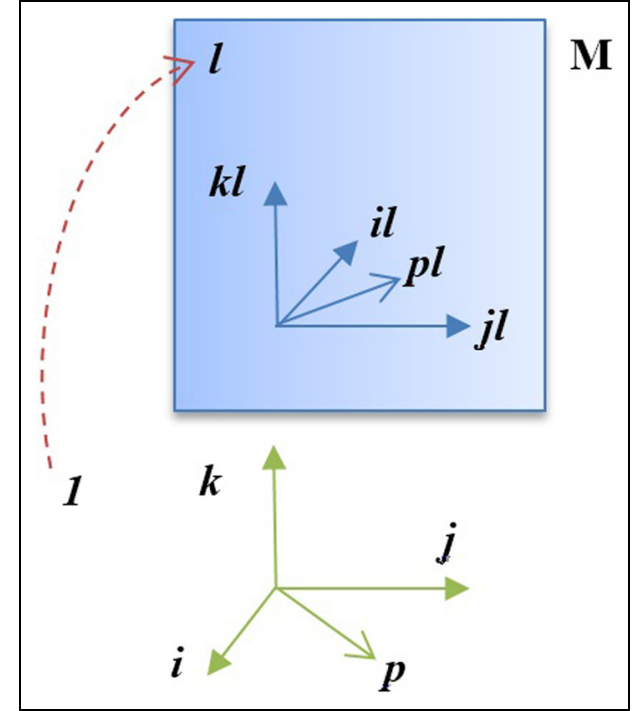

Figure 3. Octonion description by employing a mirror.

Suppose $\mathrm{Q}_{4}$ is a quaternion and its conjugate $\mathrm{Q}_{4}^{*}$. Defining an octonion $\mathrm{O}_{8}$, it is easy to find that

$$
\mathrm{Q}_{4} \mathrm{O}_{8} \mathrm{Q}_{4}^{*}=\left(\mathrm{Q}_{4} \mathrm{O}_{8}\right) \mathrm{Q}_{4}^{*}=\mathrm{Q}_{4}\left(\mathrm{O}_{8} \mathrm{Q}_{4}^{*}\right)
$$

The first four items of octonions in equation (18) are just a quaternion, which can represent a spatial position rotation in a three-dimensional (3D) space.

The octonion's space can be denoted by the quaternion's space combined with a mirror $\mathbf{M}$, as shown in Figure 3.

In Figure 3, $1, \boldsymbol{i}, \boldsymbol{j}$ and $\boldsymbol{k}$ are the four bases of quaternions (1-space), and $\boldsymbol{i l}, \boldsymbol{j l}$ and $\boldsymbol{k} \boldsymbol{l}$ are the images of $\boldsymbol{i}, \boldsymbol{j}$ and $\boldsymbol{k}$ in the mirror $\mathbf{M}$, which comply with the lefthand coordinate system. Hence, $\boldsymbol{l}, \boldsymbol{i l}, \boldsymbol{j} \boldsymbol{l}$ and $\boldsymbol{k} \boldsymbol{l}$ constitute another four-dimensional (4D) space (l-space). As a result, $\mathbf{1}, \boldsymbol{i}, \boldsymbol{j}$ and $\boldsymbol{k}$ and $\boldsymbol{l}, \boldsymbol{i} \boldsymbol{l}, \boldsymbol{j} \boldsymbol{l}$ and $\boldsymbol{k} \boldsymbol{l}$ constitute an 8D space, which are the bases of octonions. $\boldsymbol{p}$ is an arbitrary vector in the 1-space, and its image in $\mathrm{M}$ is $\boldsymbol{p l}$. Similar to pure quaternion, it can be denoted by pure octonion as $O_{P}=\boldsymbol{p}+\boldsymbol{p l}$. It should be noted that this is only one of the various descriptions for octonions suitable for this article.

\section{Rotations and octonions}

Since the octonions were discovered independently by Arthur Cayley in 1845, one of the most familiar applications is to describe the process whereby an electron emits or absorbs a photon, ${ }^{16}$ where it can be used to describe the spinor.

\section{Octonions in 3D coordinate system}

In Figure 2, the resulting vector $\boldsymbol{q}=\boldsymbol{p}_{\|}+\boldsymbol{p}_{\perp}{ }_{\perp} \cdot \boldsymbol{p}_{\|}$is not affected by the rotation, and it remains unchanged after rotation. 


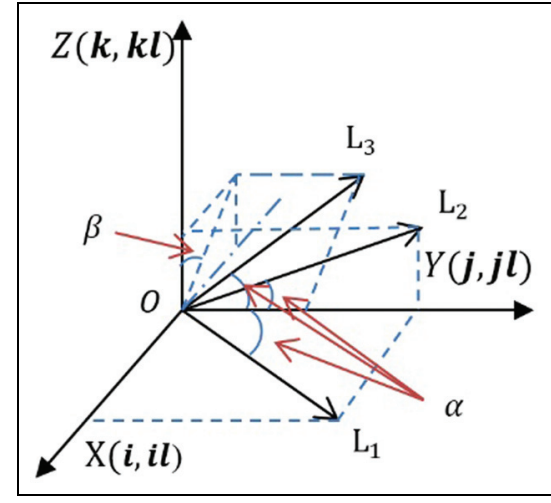

Figure 4. 3D coordinate system.

In Figure 4, assume $\mathrm{X}, \mathrm{Y}$ and $\mathrm{Z}$ axes are rotated around the $Z$ axis by an angle of $2 \theta$, then we obtain

$$
\begin{aligned}
& \mathrm{X} \text { axis : } \mathrm{O}^{\prime}{ }_{x}=\mathrm{Q}_{z} \mathrm{O}_{x} \mathrm{Q}_{z}^{*} \\
& =(\boldsymbol{i} \cos 2 \theta+\boldsymbol{j} \sin 2 \theta)+\boldsymbol{i l}(\cos 2 \theta-\boldsymbol{k} \sin 2 \theta) \\
& \mathrm{Y} \text { axis : } \mathrm{O}^{\prime}{ }_{y}=\mathrm{Q}_{z} \mathrm{O}_{y} \mathrm{Q}_{z}^{*} \\
& =(-\boldsymbol{i} \sin 2 \theta+\boldsymbol{j} \cos 2 \theta)+\boldsymbol{j} \boldsymbol{l}(\cos 2 \theta-\boldsymbol{k} \sin 2 \theta) \\
& \mathrm{Z} \text { axis : } \mathrm{O}_{z}^{\prime}=\mathrm{Q}_{z} \mathrm{O}_{z} \mathrm{Q}_{z}^{*}=\boldsymbol{k}+\boldsymbol{k} \boldsymbol{l}(\cos 2 \theta-\boldsymbol{k} \sin 2 \theta)
\end{aligned}
$$

where $\mathrm{Q}_{z}=\cos \theta+\boldsymbol{k} \sin \theta, \mathrm{Q}_{z}^{*}=\cos \theta-\boldsymbol{k} \sin \theta, \mathrm{O}_{x}=$ $\boldsymbol{i}+\boldsymbol{i l}, \mathrm{O}_{y}=\boldsymbol{j}+\boldsymbol{j} \boldsymbol{l}$ and $\mathrm{O}_{z}=\boldsymbol{k}+\boldsymbol{k} \boldsymbol{l}$.

It is obvious that $(\boldsymbol{i} \cos 2 \theta+\boldsymbol{j} \sin 2 \theta),(-\boldsymbol{i} \sin 2 \theta+$ $\boldsymbol{j} \cos 2 \theta)$ and $\boldsymbol{k}$ represent the spatial position vectors, while $\boldsymbol{i l}(\cos 2 \theta-\boldsymbol{k} \sin 2 \theta), \quad \boldsymbol{j} l(\cos 2 \theta-\boldsymbol{k} \sin 2 \theta)$ and $\boldsymbol{k} \boldsymbol{l}(\cos 2 \theta-\boldsymbol{k} \sin 2 \theta)$ describe the rotation processes.

Suppose three lines $\mathrm{L}_{1}, \mathrm{~L}_{2}$ and $\mathrm{L}_{3}$ are in the planes XOY and YOZ and the space OXYZ, respectively, with the same inclined angle $\alpha$ with $\mathrm{Y}$ axis as shown in Figure 4. The vectors of the three lines can be described as

$$
\begin{array}{ll}
\mathrm{L}_{1}: & \boldsymbol{V}_{1}=\boldsymbol{i} \sin \alpha+\boldsymbol{j} \cos \alpha \\
\mathrm{L}_{2}: & \boldsymbol{V}_{2}=\boldsymbol{j} \cos \alpha+\boldsymbol{k} \sin \alpha \\
\mathrm{L}_{3}: & \boldsymbol{V}_{3}=-\boldsymbol{i} \sin \alpha \sin \beta+\boldsymbol{k} \sin \alpha \cos \beta+\boldsymbol{j} \cos \alpha
\end{array}
$$

where $\beta$ is the angle between the $Z$ axis and the projection of $\mathrm{L}_{3}$ in the plane XOZ.

Define an octonion

$$
\mathrm{O}=\mathrm{O}_{p}+\mathrm{O}_{r}
$$

where $\mathrm{O}_{p}=\mathrm{a}_{0}+\mathrm{a}_{1} \boldsymbol{i}+\mathrm{a}_{2} \boldsymbol{j}+\mathrm{a}_{3} \boldsymbol{k}$, denoting the position vector, $\mathrm{O}_{r}=\mathrm{a}_{4} \boldsymbol{l}+\mathrm{a}_{5} \boldsymbol{i} \boldsymbol{l}+\mathrm{a}_{6} \boldsymbol{j} \boldsymbol{l}+\mathrm{a}_{7} \boldsymbol{k} \boldsymbol{l}$, denoting the rotation angle vector. Since the position vector is the same as the quaternions described above during the rotation, here we only focus on the rotation angle vector.

Suppose that $\mathrm{Y}$ axis is rotated $2 \theta$ around $\mathrm{L}_{1}, \mathrm{~L}_{2}$ and $\mathrm{L}_{3}$, respectively, then we obtain

$$
\begin{aligned}
\mathrm{L}_{1}: \mathrm{O}_{r 1} & =\mathrm{Q}_{l 1} \mathrm{O}_{r y} \mathrm{Q}_{l 1}^{*} \\
& =-\boldsymbol{l}(\sin 2 \theta \cos \alpha+\boldsymbol{j} \cos 2 \theta-\boldsymbol{k} \sin 2 \theta \sin \alpha) \\
\mathrm{L}_{2}: \mathrm{O}_{r 2} & =\mathrm{Q}_{l 2} \mathrm{O}_{r y} \mathrm{Q}_{l 2}^{*} \\
& =-\boldsymbol{l}(\sin 2 \theta \cos \alpha+\boldsymbol{j} \cos 2 \theta+\boldsymbol{i} \sin 2 \theta \sin \alpha)
\end{aligned}
$$

$$
\begin{aligned}
\mathrm{O}_{r 3} & =\mathrm{Q}_{l 3} \mathrm{O}_{r y} \mathrm{Q}_{l 3}^{*} \\
& =-\boldsymbol{l}[\sin 2 \theta \cos \alpha+\boldsymbol{j} \cos 2 \theta \\
& +(\boldsymbol{i} \sin 2 \theta \sin \alpha \cos \beta+\boldsymbol{k} \sin 2 \theta \sin \alpha \sin \beta)]
\end{aligned}
$$

where $\mathrm{Q}_{l 1}=\cos \theta+\boldsymbol{V}_{1} \sin \theta, \quad \mathrm{Q}_{l 1}^{*}=\cos \theta-\boldsymbol{V}_{1} \sin \theta$, $\mathrm{Q}_{l 2}=\cos \theta+\boldsymbol{V}_{2} \sin \theta, \quad \mathrm{Q}_{l 2}^{*}=\cos \theta-\boldsymbol{V}_{2} \sin \theta, \quad \mathrm{Q}_{l 3}=$ $\cos \theta+\boldsymbol{V}_{3} \sin \theta, \mathrm{Q}_{l 3}^{*}=\cos \theta-\boldsymbol{V}_{3} \sin \theta$ and $\mathrm{O}_{r y}=\boldsymbol{j} \boldsymbol{l}$.

The results indicate that $(\sin 2 \theta \cos \alpha)$ is a constant; $(j \cos 2 \theta)$ is the segment related to the rotated vector (Y axis); $(-\boldsymbol{k} \sin 2 \theta \sin \alpha),(\boldsymbol{i} \sin 2 \theta \sin \alpha)$ and $[(\boldsymbol{i} \cos \beta+\boldsymbol{k}$ $\sin \beta) \sin 2 \theta \sin \alpha]$ are the segments describing the perpendicular vector that complies with the left-hand coordinate system with the rotation axis and $\mathrm{Y}$ axis. Combined with equations (20), (21) and (22), the rotation process of a vector around an axis can be denoted by two rotation segments of an octonion, that is, the rotating component around a perpendicular axis and the spinor, the coefficients are invariant in different coordinate systems and are determined by the rotation quaternion.

\section{Arbitrary nonorthogonal rotation}

An arbitrary nonorthogonal rotation can be shown in Figure 5, $\overrightarrow{\mathrm{OA}}$ makes an inclined angle $\alpha(0<\alpha<\pi / 2)$ with $\mathrm{OP}$ and rotates $2 \theta(0 \leqslant 2 \theta<\pi)$ around $\mathrm{OP}$ to $\overrightarrow{\mathrm{OB}}$. Define the original vector $\mathrm{OA}$ as $\mathrm{OY} 1$ axis; $\mathrm{OY}_{0}$, $\mathrm{OY}_{1}, \mathrm{OZ}_{0}$ and $\mathrm{OZ}_{1}$ are in the same plane; $\mathrm{OZ}_{2}$ is perpendicular to $\overrightarrow{\mathrm{OA}}$ and $\overrightarrow{\mathrm{OB}} ;$ and $\mathrm{OX}_{0} \perp \mathrm{OY}_{0} \perp$ $\mathrm{OZ}_{0}, \mathrm{OX}_{0} \perp \mathrm{OY}_{1} \perp \mathrm{OZ}_{1}, \mathrm{OX}_{2} \perp \mathrm{OY}_{1} \perp \mathrm{OZ}_{2}$ and $\overrightarrow{\mathrm{AM}}$ $\perp \overrightarrow{\mathrm{OA}} \perp \mathrm{OX}_{0}, \overrightarrow{\mathrm{AM}}$ could be used to denote the spinning angle of $O A$.

In the coordinate system $\mathrm{OX}_{0} \mathrm{Y}_{1} \mathrm{Z}_{1}$, the rotation is same as the rotation around $\mathrm{L}_{2}$ in Figure 4. Take $\bar{O} A$ and $O B$ as unit vectors, the result can be described as

$$
\begin{aligned}
\mathrm{O}_{\mathrm{OB} 1} & =\mathrm{Q}_{\mathrm{OP}} \mathrm{O}_{\mathrm{OA}} \mathrm{Q}_{\mathrm{OP}}^{*} \\
& =-\boldsymbol{l} \sin 2 \theta \cos \alpha+\boldsymbol{j} \boldsymbol{l} \cos 2 \theta+\boldsymbol{i} \boldsymbol{l} \sin 2 \theta \sin \alpha
\end{aligned}
$$

where $\mathrm{Q}_{\mathrm{OP}}=\cos \theta+(\boldsymbol{j} \cos \alpha+\boldsymbol{k} \sin \alpha) \sin \theta \quad$ and $\mathrm{Q}_{\mathrm{OP}}^{*}=\cos \theta-(\boldsymbol{j} \cos \alpha+\boldsymbol{k} \sin \alpha) \sin \theta$ and $\mathrm{O}_{\mathrm{OA}}=\boldsymbol{j} \boldsymbol{l}$.

Meanwhile, in the coordinate system $\mathrm{OX}_{2} \mathrm{Y}_{1} \mathrm{Z}_{2}$, the rotation can be divided into rotating around $\mathrm{OZ}_{2}$ which represents the position rotation process and spinning around itself. As $\bar{O} A$ rotates $2 \theta$ around $\bar{O} P$, based on two rotation quaternions, $(\cos \gamma+\boldsymbol{k} \sin \gamma)$ and $(\cos \delta+\boldsymbol{j} \sin \delta)$, the combined quaternion vector is defined as 


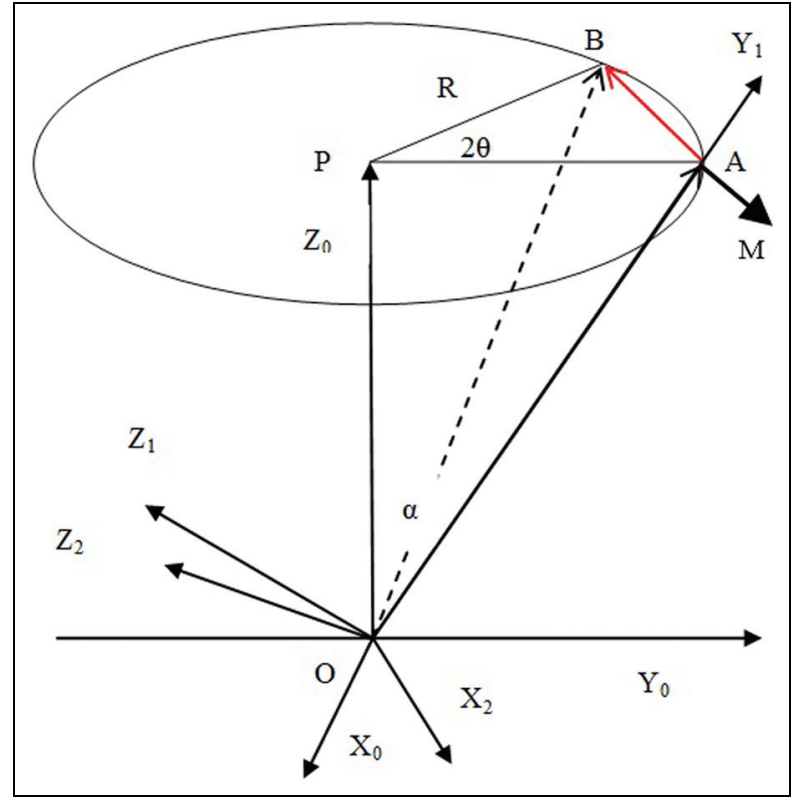

Figure 5. Arbitrary nonorthogonal rotation.

$$
\mathrm{Q}_{0}=\cos \theta+\boldsymbol{k} \sin \gamma+\boldsymbol{j} \sin \delta
$$

where $2 \gamma$ is the rotating angle (around $\mathrm{OZ}_{2}$ ) and $2 \delta$ is the spinning angle (around $\mathrm{OY}_{1}$ ) in the coordinate system $\mathrm{OX}_{2} \mathrm{Y}_{1} \mathrm{Z}_{2}$. It can be shown $\sin ^{2} \gamma+\sin ^{2} \delta=\sin ^{2} \theta$. Then, we obtain

$$
\begin{aligned}
\mathrm{O}_{\mathrm{OB} 2} & =\mathrm{Q}_{0} \mathrm{O}_{\mathrm{OA}} \mathrm{Q}_{0}^{*} \\
& =-\boldsymbol{l} 2 \sin \delta \cos \theta+\boldsymbol{j} l \cos 2 \theta+i l 2 \sin \gamma \cos \theta
\end{aligned}
$$

From $\mathrm{O}_{\mathrm{OB} 1}=\mathrm{O}_{\mathrm{OB} 2}$, we obtain

$$
\left\{\begin{array}{l}
2 \delta=2 \sin ^{-1}(\sin \theta \cos \alpha) \\
2 \gamma=2 \sin ^{-1}(\sin \theta \sin \alpha)
\end{array}\left(0 \leqslant \alpha \leqslant \frac{\pi}{2}, 0 \leqslant \theta, \delta, \gamma<\pi\right)\right.
$$

According to Figure 5, it is facile to find that $2 \gamma$ is $\angle A O B$, and $2 \delta$ is the rotation angle of $A M$ around $\bar{O} A$; since $\mathrm{OZ}_{2}$ is not parallel to $A M$, in the rotating process of $\bar{O} A$ around $\mathrm{OZ}_{2}, A M$ would produce minor rotation around $\bar{O} A$, which means $2 \delta$ is not the real spinning angle of $O A$. However, as the inclined angle of $A M$ and $\mathrm{OZ}_{2}$ is minute, this article chooses $2 \delta$ as the spinning angle of $\bar{O} A$, and the minor error is hence neglected.

\section{Calibration error analysis}

In section "Rotations and octonions," the rotation has been realized by means of decomposing the axes, compared to the decomposition of the rotated vector described in section "Quaternions and octonions." In Figure $6, \mathrm{M}_{\mathrm{N}}$ is the surface axis (surface normal) of the reflecting mirror, $M_{H}$ the horizontal axis and $M_{V}$ the vertical axis. $\mathrm{P}_{\mathrm{A}}$ and $\mathrm{P}_{\mathrm{E}}$ are the azimuth axis and the elevation axis of the platform, respectively. $T_{A}$ and $T_{E}$ are the medium axis and the outer axis of the turntable,

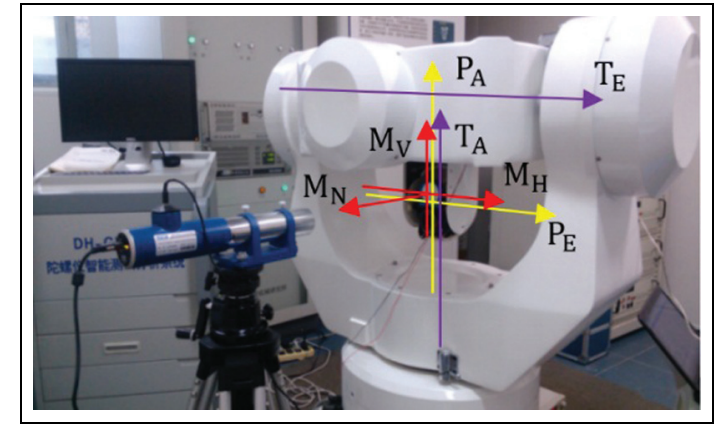

Figure 6. Experimental setup and data acquisition system.

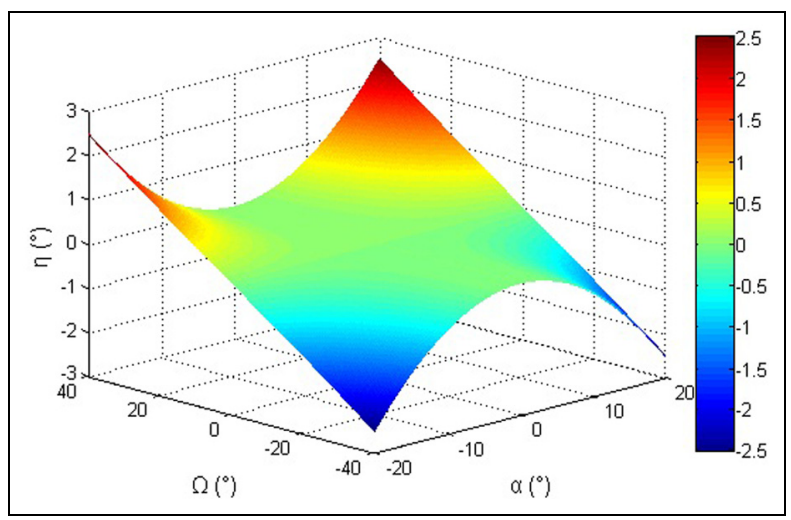

Figure 7. Calibration error's effect.

respectively. At zero positions, $\mathrm{P}_{\mathrm{A}}$ and $\mathrm{T}_{\mathrm{A}}$ are vertical, and $\mathrm{P}_{\mathrm{E}}$ and $\mathrm{T}_{\mathrm{E}}$ are horizontal.

As described in section "Introduction," the azimuth and elevation angles are defined for the platform $\left(\theta_{P A}\right.$ and $\left.\theta_{P E}\right)$, the turntable $\left(\theta_{T A}\right.$ and $\left.\theta_{T E}\right)$ and the autocollimator readouts of axes $\left(\delta_{A}\right.$ and $\left.\delta_{E}\right)$. When $\theta_{T E}=-\theta_{P E}=\alpha \neq 0$, if the turntable rotates $\Omega$ around $\mathrm{T}_{\mathrm{A}}$, the mirror will rotate at the same angle around $\mathrm{M}_{\mathrm{V}}$, if the platform continues rotating $-\Omega$ around $\mathrm{P}_{\mathrm{A}}$, according to equation (33), the mirror will only rotate $-\left[2 \sin ^{-1}(\sin (\Omega / 2) \cos \alpha)\right]$ around $\mathrm{M}_{\mathrm{V}}$ with a spinor of $-\left[2 \sin ^{-1}(\sin (\Omega / 2) \sin \alpha)\right]$ around $\mathrm{M}_{\mathrm{H}}$. The spinning of the mirror will result in the coupling error in the azimuth and elevation, but the angle is minute in this calibration process. The rotation angle will introduce an angular error $\eta$ in the azimuth

$$
\eta=\Omega-\left[2 \sin ^{-1}\left(\sin \frac{\Omega}{2} \cos \alpha\right)\right]
$$

With $\Omega$ changing from $-40^{\circ}$ to $40^{\circ}$ and $\alpha$ from $-20^{\circ}$ to $20^{\circ}$, the calibration error in simulation is shown in Figure 7. Since the calibration error significantly increases with $\Omega$ and $\alpha$, it is necessary to separate the error.

\section{Experimental results}

The pointing errors have been acquired with a highprecision turntable and an autocollimator at the 


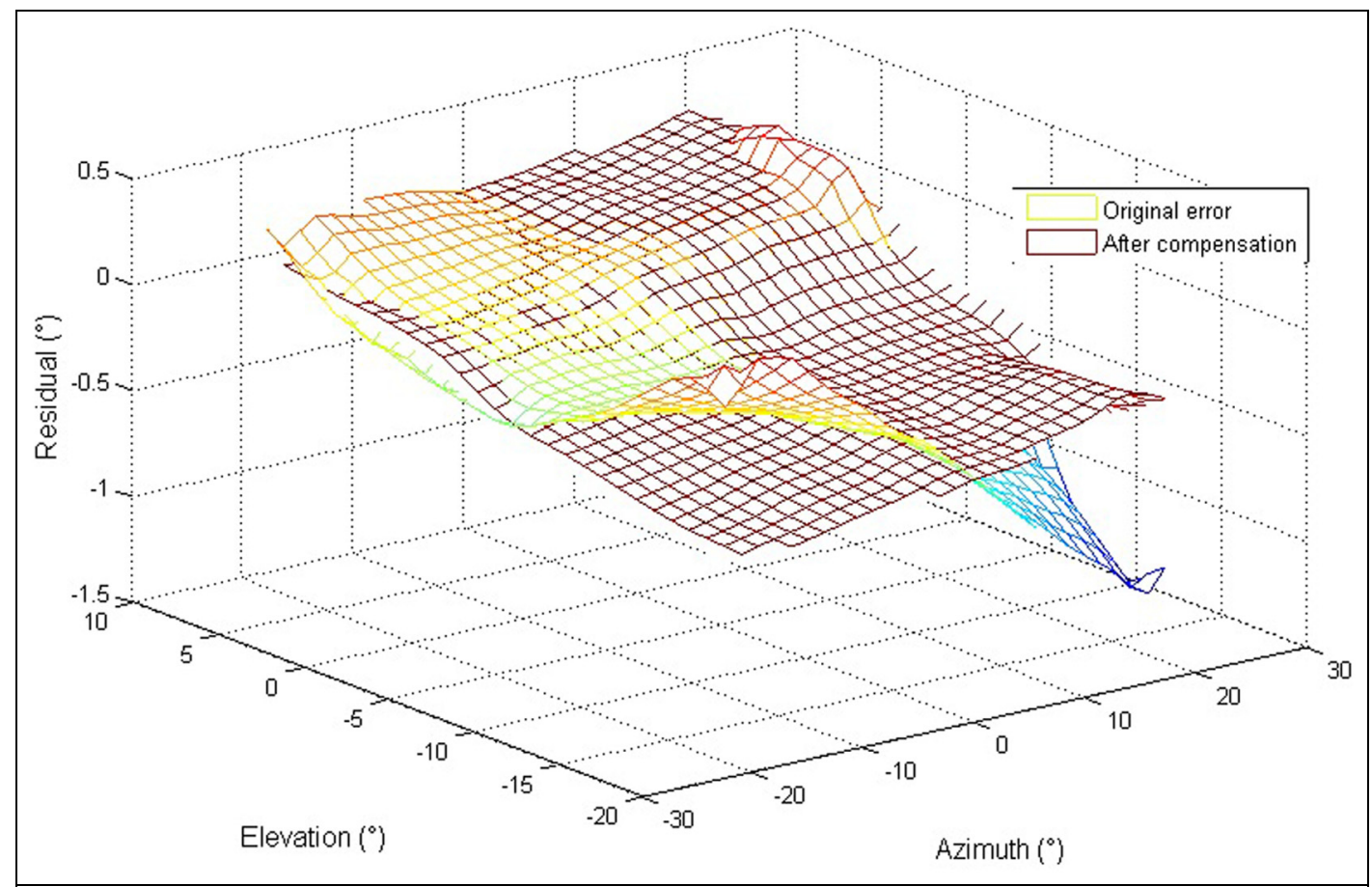

Figure 8. Azimuth error before and after compensation.

Table 2. Compensation result comparisons.

\begin{tabular}{lcc}
\hline & $\begin{array}{l}\text { Azimuth } \\
\text { (original error) }\end{array}$ & $\begin{array}{l}\text { Azimuth } \\
\text { (compensated) }\end{array}$ \\
\hline Mean value & $-0.1703^{\circ}$ & $-0.1674^{\circ}$ \\
Variance & $0.1379\left(^{\circ}\right)^{2}$ & $0.0492\left(^{\circ}\right)^{2}$ \\
Maximum value & $0.6935^{\circ}$ & $0.2496^{\circ}$ \\
Minimum value & $-1.5610^{\circ}$ & $-0.5880^{\circ}$ \\
\hline
\end{tabular}

azimuth of $-20^{\circ},-15^{\circ},-10^{\circ},-5^{\circ}, 0^{\circ}, 5^{\circ}, 10^{\circ}, 15^{\circ}$ and $20^{\circ}$ and at the elevation of $-20^{\circ},-15^{\circ},-10^{\circ},-5^{\circ}, 0^{\circ}$, $5^{\circ}$ and $10^{\circ}$. The compensation results calculated by equation (34) (the elevation angle is $\alpha$, and the azimuth angle is the rotated angle $\Omega$ ) are shown in Figure 8 and Table 2, which have shown that the calibration error has been compensated effectively. However, in order to improve its pointing accuracy further, it is necessary to analyze errors that result from the platform's misalignment error, nonperpendicularity, initialization error and so on, and then relevant compensation models can be adopted. The detailed information has been presented and reported in Hong et al. ${ }^{9}$ and Tang et al. ${ }^{17}$

\section{Conclusion}

With respect to the ISPs, calibration process with a high-precision turntable and an autocollimator, this article has developed a novel approach to the rotation decomposition to determine the calibration errors. It has shown that octonions can represent the rotation process completely, including the rotation around a space vector and the spinning around itself. It has realized a new representation of the rotation by means of decomposing the rotation axis into two perpendicular axes. The error in the calibration process is then derived. According to the test results, the accuracy after error correction is significantly improved with the variance in azimuth decreased from $0.1379\left({ }^{\circ}\right)^{2}$ to $0.0492\left({ }^{\circ}\right)^{2}$, which offers a more accurate set of data for further compensation based on the error model of the platform itself.

\section{Declaration of conflicting interests}

The author(s) disclosed receipt of the following financial support for the research, authorship, and/or publication of this article: The author(s) declared no potential conflicts of interest with respect to the research, authorship and/or publication of this article.

\section{Funding}

This work was sponsored by the Support Program of National Ministry of Education of China (no. 625010110) and the National Natural Science Foundation of China (no. 61179043).

\section{References}

1. Evans PR. Rotations and rotation matrices. Acta Crystallogr 2001; 57: 1355-1359.

2. Fillmore JP. A note on rotation matrices. IEEE Comput Graph 1984; 4: 30-33.

3. Insel AJ. Rotation matrices in the plane without trigonometry. Coll Math J 1993; 24: 71-73. 
4. Ozgoren MK. Kinematic analysis of spatial mechanical systems using exponential rotation matrices. $J$ Mech Des: T ASME 2007; 129: 1144-1152.

5. Neef J. Rotation matrices. Mach Des 1988; 60: 100-102.

6. Hilkert JM. Inertially stabilized platform technology. IEEE Contr Syst Mag 2008; 28: 26-46.

7. Michael KM. Inertially stabilized platforms for optical imaging systems. IEEE Contr Syst Mag 2008; 28: 47-64.

8. Ekstrand B. Equations of motion for a two-axes gimbal system. IEEE T Aero Elec Sys 2001; 37: 1083-1091.

9. Hong HJ, Zhou XY, Zhang ZY, et al. Modelling and calibration of pointing errors using a semi-parametric regression method with applications in inertially stabilized platforms. Proc IMechE, Part B: J Engineering Manufacture. Epub ahead of print 16 August 2013. DOI: $10.1177 / 0954405413475559$.

10. Zhang ZY, Zhou XY and Fan DP. Analysis, modelling and correction of pointing errors for electro-optical detection systems. Acta Aeronaut Astronaut Sin 2011; 32: 2042-2054.
11. Rue AK. Calibration of precision gimbaled pointing systems. IEEE T Aero Elec Sys 1970; 6: 697-706.

12. Goldman R. Understanding quaternions. Graph Model 2011; 73: 21-49.

13. Meister L and Schaeben H. A concise quaternion geometry of rotations. Math Method Appl Sci 2005; 28: $101-126$.

14. Meister L. Mathematical modelling in geodesy based on quaternion algebra. Phys Chem Earth Pt A 2000; 25: 661-665.

15. Liu JF. Three dimensional rotation represented by quaternion. College Phys 2004; 23: 39-43.

16. Baez JC. The octonions. Bull Am Math Soc 2001; 39: 145-205.

17. Tang QJ, Wang XJ and Yang Q. Static pointing error analysis of electro-optical detection systems. Proc IMechE, Part B: J Engineering Manufacture. Epub ahead of print 15 October 2014. DOI: 10.1177/ 0954405414551107. 